\title{
Utilization of Artificial Intelligence in Meeting the Challenges of COVID- 19 Pandemic: A Quick Literature Review
}

Dr. Muhammad Atif Waheed ${ }^{1 *}$, Dr. Moqthiar Shareef ${ }^{1}$, Dr. Sadaf Akbar ${ }^{2}$, Dr. Sana Arooj ${ }^{3}$, Dr. Maxwell Slieman ${ }^{2}$, Dr. Ahmed Rashid Shaik ${ }^{1}$, Dr. Hashim AlSayed Mohammed ${ }^{4}$

${ }^{1}$ Consultant Family Medicine, Primary Healthcare Corporation, Qata

${ }^{2}$ Specialist Family Medicine, Primary Healthcare Corporation, Qatar

${ }^{3}$ Junior Doctor

${ }^{4}$ Senior Consultant Family Medicine, Primary Healthcare Corporation, Qatar

DOI: $10.36347 /$ sjams.2022.v10i01.024

| Received: 18.12.2021 | Accepted: 26.01.2022 | Published: 30.01.2022

*Corresponding author: Dr. Muhammad Atif Waheed

Consultant Family Medicine, Primary Healthcare Corporation, Qatar

\section{Abstract}

The world is facing several challenges due to the COVID-19 pandemic, which is causing severe social and economic disruption. The disruption has resulted in recession, unemployment, and social isolation and has caused an extreme burden on health care services. Artificial intelligence (AI) has a promising role in the healthcare sector by bringing many advantages to practicing clinicians, patients, and society. This article aims to determine the role of artificial intelligence in the ongoing COVID-19 pandemic. A quantitative methodology is used, and a literature review is done by using electronic databases such as PubMed, Google Scholar, and Scopus. The keywords used for this data research are "artificial intelligence" and "COVID 19"; "COVID 19" and "artificial intelligence". The results have shown that artificial intelligence has been extensively used in seven major domains during the current ongoing COVID-19 pandemic. These include screening and detection of COVID-19 transmission dynamics, diagnostics, disease monitoring and forecasting, disease outbreak containment, disease recovery and mortality, treatment and vaccination, and protection of healthcare workers. Artificial intelligence is a transformational force in the medical field. It assists in early detection of disease, real-time surveillance, diagnosis, treatment, disease containment, development of treatment and vaccinations, and reducing morbidity and mortality. Artificial intelligence will help us in the future to meet many challenges in a timely fashion through the prediction of pandemics, making stakeholders worldwide well prepared to deal with epidemics and pandemics in a systematic and organized manner, avoiding economic turmoil and unnecessary morbidity and mortality.

Keywords: Artificial intelligence, healthcare challenges, COVID-19.

Copyright $\odot 2022$ The Author(s): This is an open-access article distributed under the terms of the Creative Commons Attribution 4.0 International License (CC BY-NC 4.0) which permits unrestricted use, distribution, and reproduction in any medium for non-commercial use provided the original author and source are credited.

\section{INTRODUCTION}

COVID-19 has jeopardized human life all over the world. The confirmed cases of COVID-19 up till now are $5,232,428$ with a mortality rate of 335,636 (Worldmeters, 2020). The economic and social impact of the pandemic will continue even after the pandemic is over. The whole world is looking for solutions to control COVID-19. Artificial intelligence (AI), which mimics human intelligence and augments human capabilities and functionalities, is playing a significant role in the ongoing pandemic by helping many stakeholders, such as governments, pharmacies, supply chains, banks, and healthcare professionals. It is assisting stakeholders in meeting several challenges posed by the COVID-19 pandemic at different levels, which include clinical, molecular, and societal applications (Bullock et al., 2020). It has the capability to predict the transmission dynamics of COVID-19 $(\mathrm{Hu}$ et al., 2020). Data mining algorithms can be used to determine the incidence and forecast outbreak trends (Ayyoubzadeh et al., 2020). Artificial intelligence is a powerful tool for health care planning and policymaking. However, it is argued that AI has not played a significant role in the COVID-19 pandemic yet, which is both due to a lack of data or mega data (Naudé, 2020). Hence, there is a need for extensive human AI interaction and transparency when it comes to data privacy and public health. 


\section{Methodology}

This study used a secondary qualitative research design and approach to investigate how artificial intelligence can be used to meet the challenges posed by the COVID-19 pandemic. A secondary research design was used in analysing the ways in which artificial intelligence is being developed and deployed by governments to deal with the challenges of the COVID-19 pandemic. The study's data has been collected by using electronic databases. The articles for this study were gathered from three databases, which are Google Scholar, PubMed, and Scopus. The keywords used for the search were: artificial intelligence and COVID-19; artificial intelligence and COVID 19; COVID 19 and artificial intelligence. The articles were selected based on inclusion and exclusion criteria. Although several articles have been published about the challenges presented by the COVID-19 pandemic, only relevant material has been incorporated into this paper. The research has included articles published in English only.

\section{RESULTS}

According to the literature review, AI has been used extensively in seven domains. These are summarized below in figure (1).

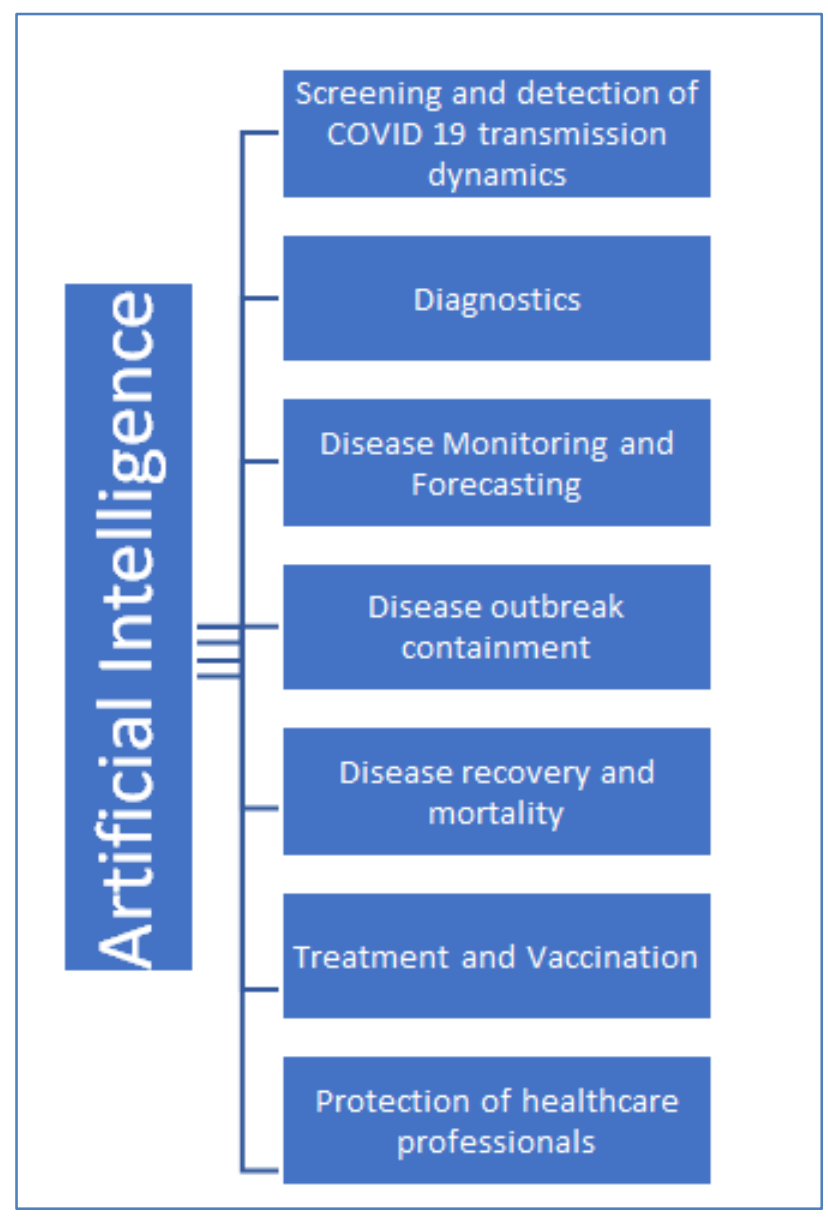

Fig-1: Summary of Artificial intelligence usage in COVID-19 pandemic

\section{1) Screening and detection of COVID 19 transmission dynamics}

AI is being used for screening, detection, prediction, and tracking of COVID-19 (Vaishya et al., 2020). Tracking and screening help to quarantine the suspects and subsequently slow down the spread of the disease. A mobile phone-based online survey which can collect recent travel history, symptoms, and signs of COVID-19 can be used for screening and early detection. The use of AI can categorize people into norisk, minimal-risk, moderate-risk, and high-risk patients. This will enable authorities to quarantine patients at an earlier stage, which will limit the spread of disease (Rao and Vazquez, 2020). It can also help authorities in taking decisions to smartly lock down areas showing an increased number of positive cases. AI-based applications assist in preparing logistics and supplies on a timely basis, thereby enabling the management of resources in a timely and systematic manner. An example of this is the development of the "Ehteraz" smartphone application by the Ministry of Interior of Qatar. The application is enabled with Global Positioning System and Bluetooth technology to spread awareness among the public regarding personal protection and to restrict outbreaks. It is useful in tracking positive cases and informs users if they have 
Muhammad Atif Waheed et al; Sch J App Med Sci, Jan, 2022; 10(1): 144-149

been in contact with positive cases. The health profile (COVID-19 status) of service users is displayed in four colors. These include red, which signifies a person with a positive COVID-19 test; yellow, a person who is in quarantine; grey, a suspected case (showing signs and symptoms) or a person who has been in contact with a positive COVID-19 person; and green, which denotes a healthy individual or a person who has tested negative for COVID-19. The colors and their signs are shown in Figure (2).

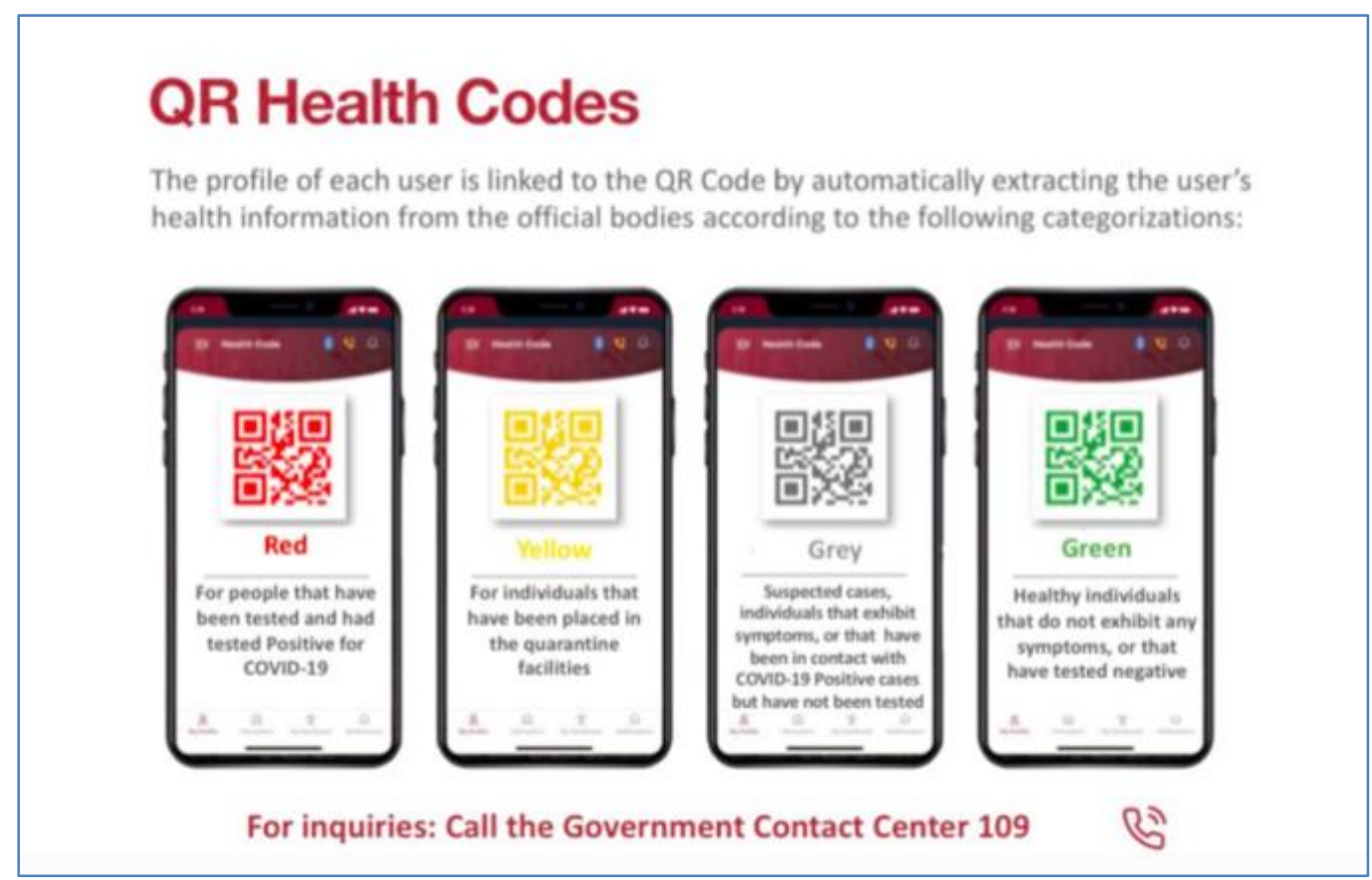

Fig-2: QR Health Codes (Qatar Day, 2020)

\section{2) Diagnostics}

With $90 \%$ accuracy, the artificial intelligencebased mobile application (AI4 COVID-19) can distinguish COVID-19 cough from other types of coughs with $90 \%$ accuracy. Greater accuracy in detection can be achieved with the availability of more data (Imran et al., 2020). Therefore, this AI-based application can be employed for disease diagnosis in the early stages. There has been tremendous pressure on radiologists to report X-rays and $\mathrm{CT}$ scans in the current COVID-19 pandemic. AI can lessen the pressure on radiologists and physicians to make prompt diagnoses. For example, AI can interpret CT images of COVID-19 patients within 20 seconds with $96 \%$ accuracy, improving diagnostic efficiency and reducing human errors (Jin et al., 2020). Ai-based software has been employed to facilitate CT diagnosis, which helps in categorizing coronaviruses according to severity level (Belfiore, 2020). Additionally, AI has been used for accelerated, scalable, and highly accurate classification of the COVID-19 genome (Randhawa et al., 2020). Recently, AI has assisted in taking real-time clinical support decisions. It was found that mildly raised ALT, myalgias, and elevated HB were most predictive of the development of ARDS with an accuracy of 70-80\% according to historical data of COVID-19 patients from two hospitals in China (Jiang et al., 2020). Hence, AI is an essential tool in augmenting physicians' roles to save lives by improving diagnostic accuracy, classifying diseases, and making evidence-based clinical decisions.

\section{3) Disease monitoring and forecasting}

Data mining can help with data collected from public health surveillance, real-time epidemic monitoring and forecasting, regular updates of situations in organizations, and real-time utilization of this information by hospitals (Bragazzi et al., 2020). AI can predict the possible emergence of coronavirus in different locations and is used for diagnosis and monitoring of COVID-19 (McCall, 2020). This not only helps in detecting vulnerable patients (high-risk groups) who are at risk of being infected but also assists in preparing logistic support for dealing with breakouts. Virtual consultations in primary and secondary care can be used to monitor these patients regularly. A deteriorating health condition and the need for psychological support can also be detected through consultations. AI tools can be developed for patient monitoring, which will help in prioritizing patients for ICU allocation (Subirana et al., 2020).

\section{4) Disease outbreak containment}

$\mathrm{AI}$ is a powerful tool that can aid in containing the spread of COVID-19 in countries such as Nigeria, where it is impossible to stop human-to-human interaction. It can also assist in mitigating the impacts of the virus and taking appropriate policy measures (Jibril and Sharif, 2020). Various data dashboards have been developed for predicting and tracking COVID-19. They give a global overview of the pandemic by enabling visualization of contagion (Naudé, 2020). An 
Muhammad Atif Waheed et al; Sch J App Med Sci, Jan, 2022; 10(1): 144-149

example of such a dashboard is that of the World Health Organization, which enables users to visualize global infected cases and mortality (Figure 3 and 4). This application portrays confirmed cases, deaths over time, and case comparisons by country, territory, and area.

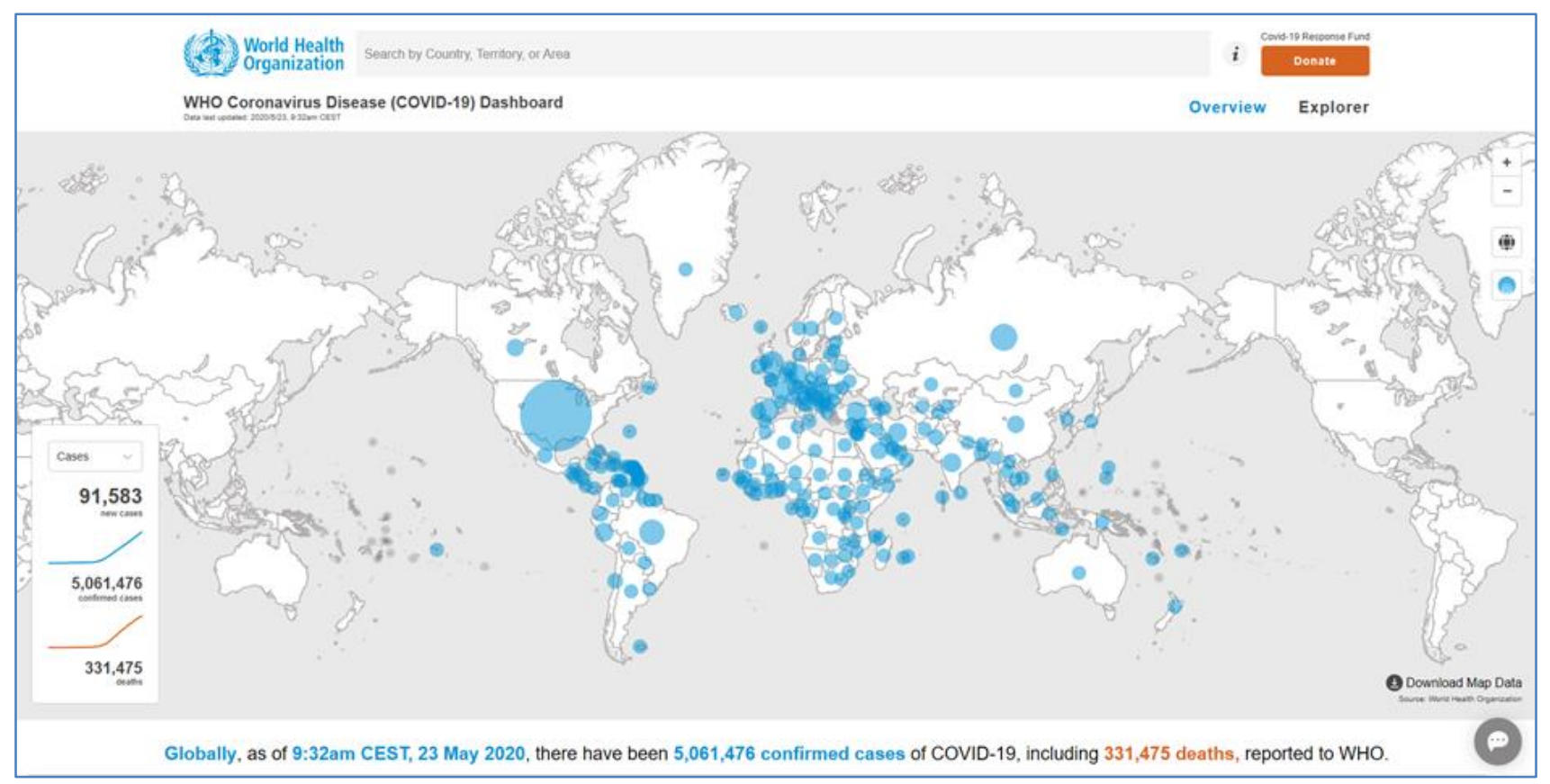

Fig-3: Global Cases (WHO, 2020)

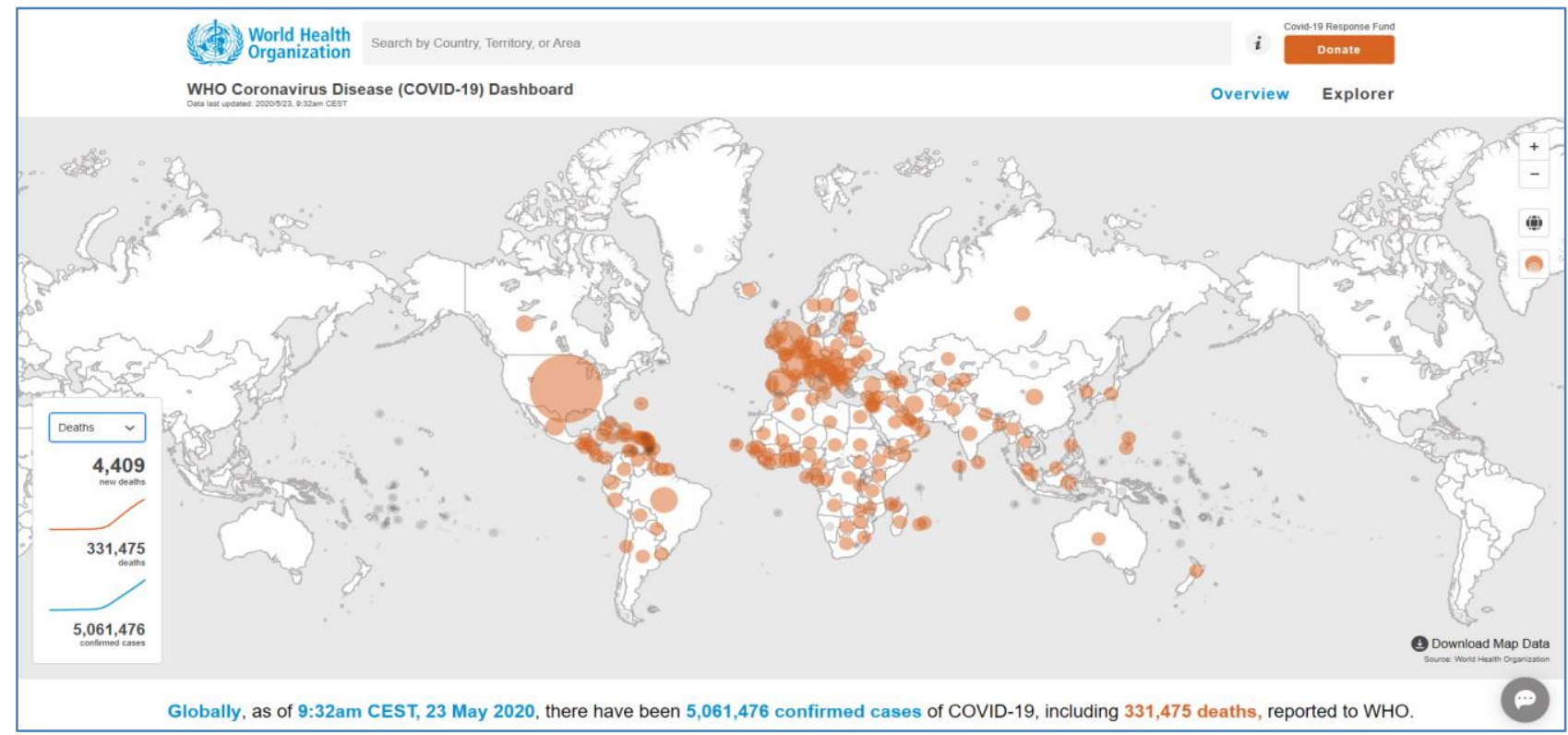

Fig-4: Global Deaths (WHO, 2020)

These dashboards have been helping governments and officials monitor the disease. Dashboards have also enabled authorities to prepare logistics in advance, such as medical workforce, quarantine centres, diagnostic equipment, and medicines. AI is also aiding in supply chain management by enabling delivery of personal protection equipment (PPE) on time and making it readily available for health care professionals.

\section{5) Disease recovery and mortality}

AI has been used to monitor patients from the beginning of infection to absolute recovery. The complete monitoring has assisted in demonstrating the clinical course of the disease. The clinical course helps to understand various factors that alter the outcome of illness, such as age, gender, and comorbidities. It has assisted in studying the epidemiological and clinical characteristics of the disease. AI is helping predict disease prognosis (Naudé, 2020). The change in lymphocyte count during the first four days of hospital 
admission has been associated with high mortality (Xie et al., 2020). AI can predict mortality by using these biomarkers. AI has been used to monitor mortality across the globe. It has helped to establish the cause of death, which could be due to multiple factors. These include demographic factors such as the elderly population, co-morbidities, or health system problems like lack of facilities and equipment. In the future, governments can use this data to prepare themselves for emergency disasters.

\section{6) Treatment and vaccination}

AI is being used for the development of treatment therapy for COVID-19 (Stebbing et al., 2020). Various trials have been going on for the treatment of COVID-19, and massive data has been collected worldwide to gauge the efficacy of treatments. Artificial intelligence is assisting researchers to find drugs that are effective against COVID-19 by incorporating real-time diagnostics techniques instead of standardized testing, which is time-consuming. Artificial intelligence has also been used in the development of vaccinations for treating COVID-19 (Vaishya et al., 2020). It is promising that a vaccine is being developed against COVID-19 at a fast pace within months, which could have taken years due to the quick processing of data. Mental health issues are on the rise during the pandemic. Artificial intelligence is helping vulnerable patients through digital mental health platforms. Similarly, for contagious dermatological diseases, hospital information systems can provide a clinical and academic platform through telemedicine and artificial intelligence (Lee et al., 2020).

\section{7) Protection of healthcare professionals}

According to a survey study in Wuhan, China, psychological stress among front-line healthcare professionals was found to be higher than college students and other medical staff residing in other cities (Wu et al., 2020). Most of the professionals are worried about getting infected themselves or transmitting it to their families. There is also the fear of being quarantined. One of the studies from Zhongnan Hospital of Wuhan University revealed that the humanto-human hospital-associated transmission of this virus is $41 \%$. The risk of transmission has been reduced using AI-enabled virtual consultations, which provide instant data on high-risk patients to the clinicians, improving their efficiency and increasing productivity (McCall, 2020). This reduces psychological distress among frontline healthcare professionals.

\section{CONCLUSION AND \\ RECOMMENDATIONS}

During the ongoing COVID-19 pandemic, artificial intelligence is playing a significant role in meeting the healthcare challenges. It plays an essential role in different domains, ranging from epidemiological monitoring, clinical course, disease classification, diagnostics, monitoring of treatment, to the development of vaccinations. With the influx of more and more data, the role of AI will be further strengthened. This will help governments, clinicians, scientists, patients, and the pharmaceutical and device industries to meet the challenges of COVID-19 through the development of tools and formulating strategies. The authorities will be able to minimize socioeconomic disruption caused by the virus and be fully prepared to combat future pandemics. Perhaps greater investment should be made in AI-based technology to develop applications to aid patients and healthcare professionals. For instance, robots should be designed to carry out swabbing and examination of COVID-19 patients, which will reduce the risk of virus transmission to healthcare professionals. Similarly, device and mobile industries can develop AI-enabled software and tools that can assist patients and doctors with various tasks. These tasks can include the recording of vital signs and patients' examinations remotely. Mobile phones with AI-enabled software can remind people about social distancing and the use of masks and gloves while leaving their homes. AIpowered mobile software should be able to monitor COVID-19 patients in quarantine and alert physicians if their health deteriorates. AI dashboards can be used to predict the financial impact of COVID-19, and governments can utilize them to develop strategies to mitigate economic risks and unemployment. The medical field is adopting technology at a swift pace and new devices with AI embedded in them are being utilized. Therefore, AI must be included in the curriculum of medical students so they can be trained and prepared to work in symbiosis with AI. Medical staff should also get training to develop the necessary skills to adapt to new AI-enabled technologies and be aware of their challenges, such as data privacy, confidentiality, and legal and ethical ramifications.

\section{Competing interests' statement} the authors.

There are no competing interests among any of

\section{Funding statement}

This research has not received any funding from any department or institute.

\section{Ethics statement}

No ethical approval was needed.

\section{REFERENCES}

- $\quad$ Ayyoubzadeh, S. M., Ayyoubzadeh, S. M., Zahedi, H., Ahmadi, M., \& Kalhori, S. R. N. (2020). Predicting COVID-19 incidence through analysis of google trends data in iran: data mining and deep learning pilot study. JMIR public health and surveillance, 6(2), e18828. 
- Belfiore, M. P., Urraro, F., Grassi, R., Giacobbe, G., Patelli, G., Cappabianca, S., \& Reginelli, A. (2020). Artificial intelligence to codify lung CT in Covid-19 patients. La radiologia medica, 125(5), 500-504.

- Bragazzi, N. L., Dai, H., Damiani, G., Behzadifar, M., Martini, M., \& Wu, J. (2020). How big data and artificial intelligence can help better manage the COVID-19 pandemic. International journal of environmental research and public health, 17(9), 3176.

- $\quad$ Bullock, J., Luccioni, A., Pham, K. H., Lam, C. S. N., \& Luengo-Oroz, M. (2020). Mapping the landscape of artificial intelligence applications against COVID-19. Journal of Artificial Intelligence Research, 69, 807-845.

- Hu, Z., Ge, Q., Li, S., Jin, L., \& Xiong, M. (2020). Artificial intelligence forecasting of covid-19 in china. arXiv preprint arXiv:2002.07112.

- Imran, A., Posokhova, I., Qureshi, H. N., Masood, U., Riaz, M. S., Ali, K., \& Nabeel, M. (2020). AI4COVID-19: AI enabled preliminary diagnosis for COVID-19 from cough samples via an app. Informatics in Medicine Unlocked, 20, 100378.

- Jiang, X., Coffee, M., Bari, A., Wang, J., Jiang, X., Huang, J., \& Huang, Y. (2020). Towards an artificial intelligence framework for data-driven prediction of coronavirus clinical severity. Computers, Materials \& Continua, 63(1), 537-551.

- Jibril, M. L., \& Sharif, U. S. (2020). Power of artificial intelligence to diagnose and prevent further covid-19 outbreak: A short communication. arXiv preprint arXiv:2004.12463.

- Jin, Y., Yang, H., Ji, W., Wu, W., Chen, S., Zhang, W., \& Duan, G. (2020). Virology, epidemiology, pathogenesis, and control of COVID19. Viruses, 12(4), 372.

- Lee, C. H. (2020). Role of dermatologists in the uprising of the novel corona virus (COVID-19): Perspectives and opportunities. Dermatologica Sinica, 38(1), 1.

- McCall, B. (2020). COVID-19 and artificial intelligence: protecting health-care workers and curbing the spread. The Lancet Digital Health, 2(4), e166-e167.
- Naudé, W. (2020). Artificial intelligence vs COVID-19: limitations, constraints and pitfalls. $A I$ \& society, 35(3), 761-765.

- Randhawa, G., Soltysiak, M., El Roz, H., de Souza, C., Hill, K. and Kari, L., 2020. Machine learning using intrinsic genomic signatures for rapid classification of novel pathogens: COVID-19 case study. PLOS ONE, 15(4), p.e0232391.

- Rao, A. S. S., \& Vazquez, J. A. (2020). Identification of COVID-19 can be quicker through artificial intelligence framework using a mobile phone-based survey when cities and towns are under quarantine. Infection Control \& Hospital Epidemiology, 41(7), 826-830.

- Stebbing, J., Phelan, A., Griffin, I., Tucker, C., Oechsle, O., Smith, D. and Richardson, P., 2020. COVID-19: combining antiviral and antiinflammatory treatments. The Lancet Infectious Diseases, 20(4), pp.400-402.

- Subirana, B., Hueto, F., Rajasekaran, P., Laguarta, J., Puig, S., Malvehy, J., \& Sarma, S. (2020). Hi sigma, do i have the coronavirus?: Call for a new artificial intelligence approach to support health care professionals dealing with the covid-19 pandemic. arXiv preprint arXiv:2004.06510.

- Vaishya, R., Javaid, M., Khan, I. H., \& Haleem, A. (2020). Artificial Intelligence (AI) applications for COVID-19 pandemic. Diabetes \& Metabolic Syndrome: Clinical Research \& Reviews, 14(4), 337-339.

- WHO, (2020). WHO Coronavirus Disease (COVID-19) Dashboard. Retrieved 23 ${ }^{\text {rd }}$ May 2020, from https://covid19.who.int/.

- Worldmeters. (2020). COVID Live - Coronavirus Statistics - Worldometer. Retrieved 23rd May 2020, from https://www.worldometers.info/coronavirus/

- Wu, W., Zhang, Y., Wang, P., Zhang, L., Wang, G., Lei, G., \& Luo, M. (2020). Psychological stress of medical staffs during outbreak of COVID-19 and adjustment strategy. Journal of medical virology, 92(10), 1962-1970.

- Xie, J., Tong, Z., Guan, X., Du, B., Qiu, H., \& Slutsky, A. S. (2020). Critical care crisis and some recommendations during the COVID-19 epidemic in China. Intensive care medicine, 46(5), 837-840. 